\title{
Front Matter: Volume 8014
}

, "Front Matter: Volume 8014," Proc. SPIE 8014, Infrared Imaging Systems: Design, Analysis, Modeling, and Testing XXII, 801401 (2 June 2011); doi: $10.1117 / 12.901330$

SPIE Event: SPIE Defense, Security, and Sensing, 2011, Orlando, Florida, United SPIE. States 


\section{PROCEEDINGS OF SPIE}

\section{Infrared Imaging Systems: Design, Analysis, Modeling, and Testing XXII}

Gerald C. Holst

Keith A. Krapels

Editors

26-28 April 2011

Orlando, Florida, United States

Sponsored and Published by

SPIE

Volume 8014

Proceedings of SPIE, 0277-786X, v. 8014 
The papers included in this volume were part of the technical conference cited on the cover and title page. Papers were selected and subject to review by the editors and conference program committee. Some conference presentations may not be available for publication. The papers published in these proceedings reflect the work and thoughts of the authors and are published herein as submitted. The publisher is not responsible for the validity of the information or for any outcomes resulting from reliance thereon.

Please use the following format to cite material from this book:

Author(s), "Title of Paper," in Infrared Imaging Systems: Design, Analysis, Modeling, and Testing XXII, edited by Gerald C. Holst, Keith A. Krapels, Proceedings of SPIE Vol. 8014 (SPIE, Bellingham, WA, 2011) Article CID Number.

ISSN 0277-786X

ISBN 9780819485885

Published by

SPIE

P.O. Box 10, Bellingham, Washington 98227-0010 USA

Telephone +1 3606763290 (Pacific Time) · Fax +1 3606471445

SPIE.org

Copyright (C) 2011, Society of Photo-Optical Instrumentation Engineers

Copying of material in this book for internal or personal use, or for the internal or personal use of specific clients, beyond the fair use provisions granted by the U.S. Copyright Law is authorized by SPIE subject to payment of copying fees. The Transactional Reporting Service base fee for this volume is $\$ 18.00$ per article (or portion thereof), which should be paid directly to the Copyright Clearance Center (CCC), 222 Rosewood Drive, Danvers, MA 01923. Payment may also be made electronically through CCC Online at copyright.com. Other copying for republication, resale, advertising or promotion, or any form of systematic or multiple reproduction of any material in this book is prohibited except with permission in writing from the publisher. The CCC fee code is 0277-786X/11/ $\$ 18.00$.

Printed in the United States of America.

Publication of record for individual papers is online in the SPIE Digital Library.

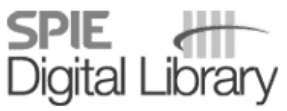

SPIEDigitallibrary.org

Paper Numbering: Proceedings of SPIE follow an e-First publication model, with papers published first online and then in print and on CD-ROM. Papers are published as they are submitted and meet publication criteria. A unique, consistent, permanent citation identifier (CID) number is assigned to each article at the time of the first publication. Utilization of CIDs allows articles to be fully citable as soon as they are published online, and connects the same identifier to all online, print, and electronic versions of the publication. SPIE uses a six-digit CID article numbering system in which:

- The first four digits correspond to the SPIE volume number.

- The last two digits indicate publication order within the volume using a Base 36 numbering system employing both numerals and letters. These two-number sets start with $00,01,02,03,04,05$, $06,07,08,09,0 A, 0 B \ldots 0 Z$, followed by 10-1Z, 20-2Z, etc.

The CID number appears on each page of the manuscript. The complete citation is used on the first page, and an abbreviated version on subsequent pages. Numbers in the index correspond to the last two digits of the six-digit CID number. 


\section{Contents}

ix Conference Committee

Workshop: New NVESD Performance Model Interface and Theory Updates

B. Teaney, J. Reynolds, U. S. Army Night Vision \& Electronic Sensors Directorate (United States)

(Presentation-Only)

\section{MODELING NON-THERMAL IMAGING SYSTEMS}

801402 Modeling of pixel-edge effects in a novel micro-filter array for the visible spectrum [8014-01] F. E. Strömqvist Vetelino, A. A. Abtahi, Aerospace Missions Corp. (United States); P. B. Griffin, Stanford Univ. (United States); R. J. Morgan, U. Raghuram, Aerospace Missions Corp. (United States)

801403 Conspicuity of moving soldiers [8014-02]

J. A. Beintema, A. Toet, S. J. de Vries, TNO (Netherlands)

801404 Modeling human performance with low light sparse color imagers [8014-03]

D. P. Haefner, J. P. Reynolds, J. Cha, V. Hodgkin, U.S. Army Night Vision \& Electronic Sensors

Directorate (United States)

801406 Improved noise model for the US Army sensor performance metric [8014-51]

B. L. Preece, J. T. Olson, J. P. Reynolds, J. D. Fanning, U.S. Army RDECOM CERDEC Night Vision

\& Electronic Sensors Directorate (United States)

801407 Passive SWIR airglow illuminated imaging compared with NIR-visible for low-light nighttime observations [8014-05]

D. C. Dayton, J. Allen, R. Nolasco, Applied Technology Associates (United States);

J. D. Gonglewski, M. Myers, Air Force Research Lab. (United States); D. Burns, I. Mons, F. Maia, Textron Defense Systems (United States)

\section{MODELING THERMAL IMAGING SYSTEMS I}

801408 Modeling MRT for well-characterized thermal imagers [8014-06]

S. D. Burks, J. P. Reynolds, K. Garner, U.S. Army RDECOM CERDEC Night Vision \& Electronic Sensors Directorate (United States)

801409 Performance evaluation of panoramic electro-optic imagers using the TOD method [8014-07]

P. Désaulniers, S. Thibault, Univ. Laval (Canada)

$80140 \mathrm{~A}$ Thermal imager non-uniformity sources modeling [8014-08]

E. Guadagnoli, C. Giunti, SELEX Galileo S.p.A. (Italy); P. Mariani, ALTRAN Italy S.p.A. (Italy); M. Olivieri, A. Porta, B. Sozzi, S. Zatti, SELEX Galileo S.p.A. (Italy) 
8014 OB Matched filtering determines human visual search in natural images [8014-09]

A. Toet, TNO (Netherlands)

8014 OC Analytical calculation for probability of detection given time-dependent search parametes [8014-10]

M. H. Friedman, J. Reynolds, D. Wilson, R. Driggers, U.S. Army RDECOM CERDEC Night Vision \& Electronic Sensors Directorate (United States)

8014 OD Drift-insensitive dim-target detection using differential correlation [8014-11]

A. Y. Hsu, Sandia National Labs. (United States)

$8014 \mathrm{OE} \quad$ Software toolkit for evaluating infrared imaging seeker [8014-12]

M. A. C. Degache, TNO Defence, Security and Safety (Netherlands)

MODELING THERMAL IMAGING SYSTEMS III

8014 OF Collaborative search with independent sensors and multiple observers [8014-13]

M. H. Friedman, J. Reynolds, U.S. Army RDECOM CERDEC Night Vision \& Electronic Sensors

Directorate (United States)

8014 OG LWIR thermal imaging through dust obscuration [8014-14]

F. A. Smith, E. L. Jacobs, S. Chari, J. Brooks, The Univ. of Memphis (United States)

\section{MODELING THERMAL IMAGING SYSTEMS IV}

$8014 \mathrm{OH} \quad$ Performance assessment of treating aliased signal as target-dependent noise [8014-15] B. L. Preece, D. P. Haefner, U.S. Army RDECOM CERDEC Night Vision \& Electronic Sensors Directorate (United States)

8014 Ol Identification of human activities in a thermal system with noise varied in temporal frequency [8014-16]

J. Brooks, E. Jacobs, F. Smith, The Univ. of Memphis (United States)

$80140 \mathrm{~J} \quad$ System considerations of aerial infrared imaging for wide-area persistent surveillance [8014-17]

M. R. Kruer, J. N. Lee, D. Linne von Berg, J. G. Howard, J. Edelberg, U.S. Naval Research Lab. (United States)

8014 OL TOD to TTP calibration [8014-19]

P. Bijl, TNO Behavioural \& Societal Sciences (Netherlands); J. P. Reynolds, U.S. Army Night Vision \& Electronic Sensors Directorate (United States); W. K. Vos, M. A. Hogervorst, TNO Behavioural \& Societal Sciences (Netherlands); J. D. Fanning, TNO Behavioural \& Societal Sciences (Netherlands) and U.S. Army Night Vision \& Electronic Sensors Directorate (United States) 
$80140 \mathrm{M}$ Spatial frequency dependence of target signature for infrared performance modeling [8014-20]

T. Du Bosq, J. Olson, U.S. Army RDECOM CERDEC Night Vision \& Electronic Sensors Directorate (United States)

$80140 \mathrm{~N} \quad$ A new blur kernel estimator and comparisons to state-of-the-art [8014-21]

L. N. Smith, J. R. Waterman, K. P. Judd, U. S. Naval Research Lab. (United States)

8014 OP Comparison of the performance of LWIR and MWIR thermal imagers for varying ambient temperature and humidity conditions [8014-23]

V. Dhar, Z. Khan, R. K. Sharma, R. Muralidharan, Solid State Physics Lab. (India)

TARGETS, BACKGROUNDS, AND ATMOSPHERICS I

$80140 Q \quad$ Improved signature prediction through coupling of ShiplR and CFD [8014-24]

D. A. Vaitekunas, W. R. Davis Engineering, Ltd. (Canada); C. Sideroff, Pointwise Inc. (United States); C. Moussa, W. R. Davis Engineering, Ltd. (Canada)

8014 OR Simulation of laser beam reflection at the sea surface [8014-25]

F. Schwenger, E. Repasi, Fraunhofer-Institut für Optronik, Systemtechnik und Bildauswertung (Germany)

8014 OS SMART and SMARTI: visible and IR atmospheric radiative-transfer libraries optimized for wide-band applications [8014-26]

V. Ross, AEREX Avionique Inc. (Canada); D. Dion, Defence Research and Development

Canada Valcartier(Canada)

8014 OT Simulation of a laser range-gated SWIR imaging system in weak turbulence conditions [8014-27]

D. Oxford, Defence Science \& Technology Lab. (United Kingdom); R. L. Espinola, U. S. Army RDECOM CERDEC Night Vision \& Electronic Sensors Directorate (United States)

$80140 \mathrm{OU}$ Computer simulation of image degradations by atmospheric turbulence for horizontal views [8014-28]

E. Repasi, R. Weiss, Fraunhofer-Institut für Optronik, Systemtechnik und Bildauswertung (Germany)

8014 OV Spatial and temporal variability of SWIR air glow measurements [8014-29]

J. Allan, D. Dayton, Applied Technology Associates (United States); J. Gonglewski, M. Myers, Air Force Research Lab. (United States); R. Nolasco, Applied Technology Associates (United States)

TARGETS, BACKGROUNDS, AND ATMOSPHERICS II

8014 OW Statistics of the point spread function for imaging through turbulence [8014-30]

M. Charnotskii, Zel Technologies, LLC and NOAA/Earth System Research Lab. (United States) 
8014 OX CART V: recent advancements in computer-aided camouflage assessment [8014-31] T. Müller, M. Müller, Fraunhofer-Institut für Optronik, Systemtechnik und Bildauswertung (Germany)

8014 OY A simple physical model for simulating turbulent imaging [8014-32]

G. Potvin, J. L. Forand, D. Dion, Defence Research and Development Canada Valcartier(Canada)

$80140 Z$ MATISSE-v2.0: new functionalities and comparison with MODIS satellite images [8014-33] L. Labarre, K. Caillault, S. Fauqueux, C. Malherbe, A. Roblin, B. Rosier, P. Simoneau, ONERA (France); C. Schweitzer, K. Stein, N. Wendelstein, Fraunhofer-Institut für Optronik, Systemtechnik und Bildauswertung (Germany)

TESTING I

801410 3D detector noise revisited [8014-34]

A. Lundmark, Autoliv Electronics AB (Sweden)

$801411 \quad$ MR-i dual-band MWIR imaging FTS [8014-35]

F. Prel, L. Moreau, S. Lantagne, ABB Analytical Measurement (Canada); R. D. Bullis, Naval Air Warfare Ctr. Aircraft Div. (United States); C. Roy, C. Vallières, L. Levesque, ABB Analytical Measurement (Canada)

801412 Design and characterization of uniform radiance source systems for veiling glare testing of optical systems via the integral method [8014-36]

J. W. Jablonski, C. Durell, G. A. McKee, Labsphere, Inc. (United States)

801413 A unique, accurate LWIR optics measurement system [8014-37]

S. D. Fantone, D. G. Orband, Optikos Corp. (United States)

$801414 \quad$ Blackbody source technology trends [8014-38]

J. A. Mazzetta, M. A. Medina, S. D. Scoptaz, J. E. Sgheiza, Electro Optical Industries, Inc. (United States)

801415 Calibration of a high dynamic range, low light level visible source [8014-39]

J. LaVeigne, T. Szarlan, N. Radtke, Santa Barbara Infrared, Inc. (United States)

TESTING II

801416 Removing ths statistical bias from three-dimensional noise measurements [8014-40] Z. Bomzon, Cl Systems (Israel) Ltd. (Israel)

801417 A common architecture for TPS development [8014-41]

A. Irwin, J. Laveigne, B. Nehring, Santa Barbara Infrared, Inc. (United States)

801418 SR-5000N design: spectroradiometer's new performance improvements in FOV response uniformity (flatness) scan speed and other important features [8014-42]

D. Cabib, S. Shapira, M. Lavi, A. Gil, U. Milman, CI Systems Ltd. (Israel) 
801419 Increased responsivity pyroelectric radiometer with dome input and temperature control [8014-43]

G. P. Eppeldaver, J. Zeng, L. M. Hanssen, National Institute of Standards and Technology (United States)

$80141 \mathrm{~A}$ Fast MTF and aberrations analysis of MWIR and LWIR imaging systems using quadri-wave interferometry [8014-44]

S. Velghe, E. Durand, D. Brahmi, W. Boucher, B. Wattellier, PHASICS S.A. (France)

\section{POSTER SESSION}

8014 1B Dual-wavelength transfer standard for laser peak-power measurement [8014-45]

R. Leonhardt, National Institute of Standards and Technology (United States); D. King, Naval Surface Warfare Ctr. Corona Div. (United States)

$80141 \mathrm{C}$ Field performance evaluation for Heliborne FLIR systems by newly devised conversion methodology [8014-46]

K. T. Han, Agency for Defense Development (Korea, Republic of)

$80141 \mathrm{E} \quad$ Laboratory for testing electro-optical surveillance systems [8014-48]

K. Chrzanowski, Military Univ. of Technology (Poland) and INFRAMET (Poland)

8014 IG High brightness imaging system using vertical cavity surface-emitting laser micro-arraysresults and proposed enhancements [8014-50]

M. A. Mentzer, U.S. Army Research Lab. (United States); C. L. Ghosh, Princeton Optronics, Inc. (United States)

Author Index 
Downloaded From: https://www.spiedigitallibrary.org/conference-proceedings-of-spie on 25 Apr 2023

Terms of Use: https://www.spiedigitallibrary.org/terms-of-use 


\title{
Conference Committee
}

\author{
Symposium Chair
}

William Jeffrey, HRL Laboratories, LLC (United States)

Symposium Cochair

Kevin P. Meiners, Office of the Secretary of Defense (United States)

Conference Chairs

Gerald C. Holst, JCD Publishing (United States)

Keith A. Krapels, U.S. Army Night Vision \& Electronic Sensors Directorate (United States)

Program Committee

Piet Bijl, TNO Defence, Security and Safety (Netherlands)

Ronald G. Driggers, U.S. Naval Research Laboratory (United States)

Richard L. Espinola, U.S. Army Night Vision \& Electronic Sensors

Directorate (United States)

David P. Forrai, L-3 Communications Cincinnati Electronics (United States)

Terrence S. Lomheim, The Aerospace Corporation (United States)

Alan Irwin, Santa Barbara Infrared, Inc. (United States)

Hector Reyes, Raytheon Company (United States)

Endre Repasi, Fraunhofer-Institut für Optronik, Systemtechnik und Bildauswertung (Germany)

Joseph P. Reynolds, U.S. Army Night Vision \& Electronic Sensors

Directorate (United States)

Bernard M. Rosier, ONERA (France)

Ronald B. Sartain, U.S. Army Research Laboratory (United States)

Michael A. Soel, FLIR Systems, Inc. (United States)

Curtis M. Webb, Northrop Grumman Corporation (United States)

Session Chairs

$1 \quad$ Modeling Non-Thermal Imaging Systems

Ronald G. Driggers, U.S. Naval Research Laboratory (United States)

Keith A. Krapels, U.S. Army Night Vision \& Electronic Sensors Directorate (United States)

Terrence S. Lomheim, The Aerospace Corporation (United States) 
$2 \quad$ Modeling Thermal Imaging Systems I

Ronald G. Driggers, U.S. Naval Research Laboratory (United States)

Keith A. Krapels, U.S. Army Night Vision \& Electronic Sensors Directorate (United States)

Terrence S. Lomheim, The Aerospace Corporation (United States)

3 Modeling Thermal Imaging Systems II

Hector Reyes, Raytheon Company (United States)

Joseph P. Reynolds, U.S. Army Night Vision \& Electronic Sensors Directorate (United States)

Piet Bijl, TNO Defence, Security and Safety (Netherlands)

$4 \quad$ Modeling Thermal Imaging Systems III

Hector Reyes, Raytheon Company (United States)

Joseph P. Reynolds, U.S. Army Night Vision \& Electronic Sensors Directorate (United States)

Piet Bijl, TNO Defence, Security and Safety (Netherlands)

5 Joint Keynote Session with Conference 8012

Paul R. Norton, U.S. Army Night Vision \& Electronic Sensors Directorate (United States)

$6 \quad$ Modeling Thermal Imaging Systems IV

Ronald B. Sartain, U.S. Army Research Laboratory (United States)

$7 \quad$ Modeling Thermal Imaging Systems $V$

Ronald B. Sartain, U.S. Army Research Laboratory (United States)

8 Targets, Backgrounds, and Atmospherics I

Endre Repasi, Fraunhofer-Institut für Optronik, Systemtechnik und Bildauswertung (Germany)

Bernard M. Rosier, ONERA (France)

Richard L. Espinola, U.S. Army Night Vision \& Electronic Sensors

Directorate (United States)

9 Targets, Backgrounds, and Atmospherics II

Endre Repasi, Fraunhofer-Institut für Optronik, Systemtechnik und Bildauswertung (Germany)

Bernard M. Rosier, ONERA (France)

Richard L. Espinola, U.S. Army Night Vision \& Electronic Sensors Directorate (United States)

10 Testing I

Alan Irwin, Santa Barbara Infrared, Inc. (United States)

Curtis M. Webb, Northrop Grumman Electronic Systems (United States) 
11 Testing II

Alan Irwin, Santa Barbara Infrared, Inc. (United States)

Curtis M. Webb, Northrop Grumman Electronic Systems (United States)

Downloaded From: https://www.spiedigitallibrary.org/conference-proceedings-of-spie on 25 Apr 2023 Terms of Use: https://www.spiedigitallibrary.org/terms-of-use 
Downloaded From: https://www.spiedigitallibrary.org/conference-proceedings-of-spie on 25 Apr 2023

Terms of Use: https://www.spiedigitallibrary.org/terms-of-use 